\title{
Structural Characterization of Aluminum Oxidation due to Exposure to Hyperthermal Atomic Oxygen
}

\author{
Long Li*, Timothy K. Minton** and Judith C. Yang* \\ *Department of Materials Science and Engineering, University of Pittsburgh, Pittsburgh, PA 15261. \\ **Department of Chemistry and Biochemistry, Montana State University, Bozeman, MT 59717.
}

Hyper-thermal atomic oxygen (AO) is the primary species in the Low Earth Orbit (from 200 to 700 $\mathrm{km}$ above the earth), hastening premature failure to materials. We studied AO oxidation of aluminum since aluminum and its alloys are used as structural components on space vehicles, as well as coating material to enhance reflectivity $[1,2]$. $\mathrm{Al}(100)$ single crystal was maintained at $220^{\circ} \mathrm{C}$ and exposed to a $5 \mathrm{eV}$ AO beam with a total fluence of $8 \times 10^{19}$ atom $\cdot \mathrm{cm}^{-2}$. The AO beam for the exposure of samples was created by detonation of $\mathrm{O}_{2}$ with a pulsed $\mathrm{CO}_{2}$ laser. Cross-sectional transmission electron microscope (TEM) samples were prepared that could be examined in both TEM and scanning electron microscope (SEM). For observations of the growth of the oxide scale, $\mathrm{Al}(100)$ single crystal was thinned by $\mathrm{Ar}^{+}$milling at low angles, and then exposed to the $5 \mathrm{eV} \mathrm{AO}$ source at room temperature for incremental time periods and taken out of the AO chamber for characterization by ex situ TEM and SEM in order to observe the evolution of the oxide scale.

Fig. 1 is images of structural investigations of the cross-sectional samples of $\mathrm{Al}(100)$ oxidized at $220^{\circ} \mathrm{C}$ with a fluence of $8 \times 10^{19}$ atom $\cdot \mathrm{cm}^{-2}$. The SEM image on Fig. 1 (a) reveals that two types of interfaces: smooth (noted by arrows) and rough areas, which exist along the interface of the oxide scale/Al(100) substrate. The smooth area shows a thin, uniform oxide layer on the Al crystal. The rough interface may be formed from the imperfect area of Al substrate, where AO diffuses through oxide layer to react with Al. The high-resolution TEM (HRTEM) image shown in Fig. 1(b) reveals that the AO creates a rough interface, where oxide/metal interfaces along Al(111) preferentially formed. Fig. 1(c) and (d) are HRTEM and high-angle annular dark field images from the same area of the sample, revealing a uniform amorphous oxide layer with a thickness of $5.7 \mathrm{~nm}$.

Fig. 2. is a set of SEM images of the $\mathrm{Al}(100)$ oxidized in $\mathrm{AO}$ at room tempera-ture as a function of exposure time. The AO exposure time was 5.5, 60 and 230 minutes with corresponding fluences of $6 \times 10^{17}, 6.6 \times 10^{18}$ and $2.5 \times 10^{19}$ atom $\cdot \mathrm{cm}^{-2}$, respectively. Fig. 2. (a) is an image of the Al(100) surface before exposure to AO. The large, flat terraces were produced by low angle $\mathrm{Ar}^{+}$milling. After 5 minutes of AO exposure, a severe surface roughness was produced (see Fig. 2. (b) and its magnified image on Fig. 2. (e)). The unusual surface roughness relates to an intriguing initial mass loss, which requires further studies. Upon further AO exposure, the surface became smoother (Fig. 2. c and d ). TEM studies reveals a thin, amorphous alumina layer developing on the surface of $\mathrm{Al}(100)$.

\section{References}

[1] G. A. Adegboyega, Journal de Physique III 2 (9), 1749 (1992).

[2] L. Li, L. Wang, T. K. Minton and J. C. Yang, Mat. Res. Soc. Symp. Proc. NN9.4, V851 (2005).

[3] This Multi-University Research Initiative (MURI) is supported by the Air Force Office of

Scientific Research under grant F49620-01-1-0336. The characterization was performed in the

Center for Microanalysis of Materials, University of Illinois at Urbana-Champaign, which is 
partially supported by the U.S. Department of Energy under grant DEFG02-96-ER45439. Authors thank Dr. R. Twesten, Dr. J.G. Wen, Dr. V. Petrova, A.M. Stewart and C.V. Ormer for their help.

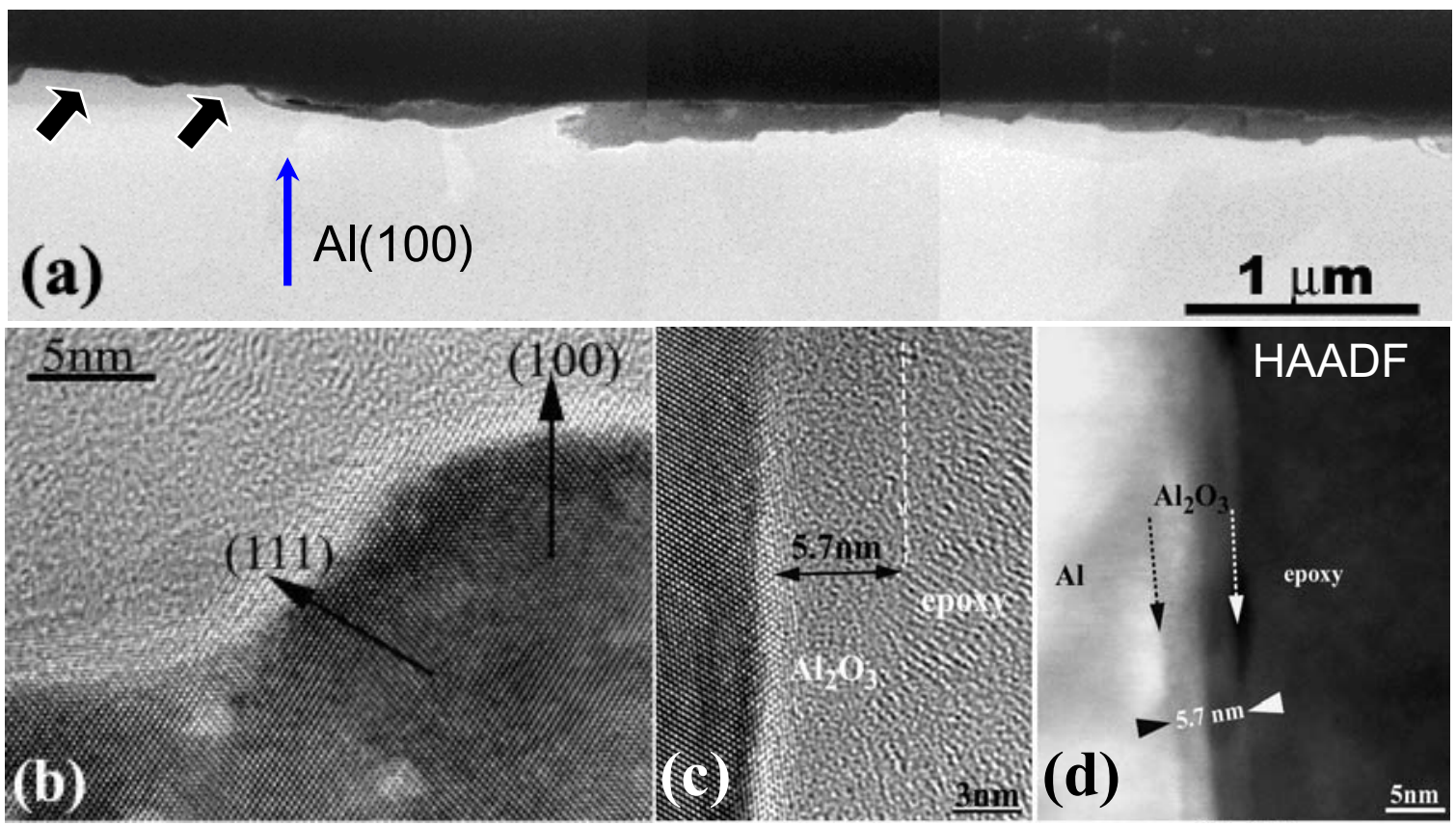

Fig. 1. Cross-sectional structural investigations of the oxide scale that formed on $\mathrm{Al}(100)$ at $220^{\circ} \mathrm{C}$. (a) SEM image of the interface, (b) and (c) HR-TEM images, and (d) high-angle annular dark-field (HAADF) image.

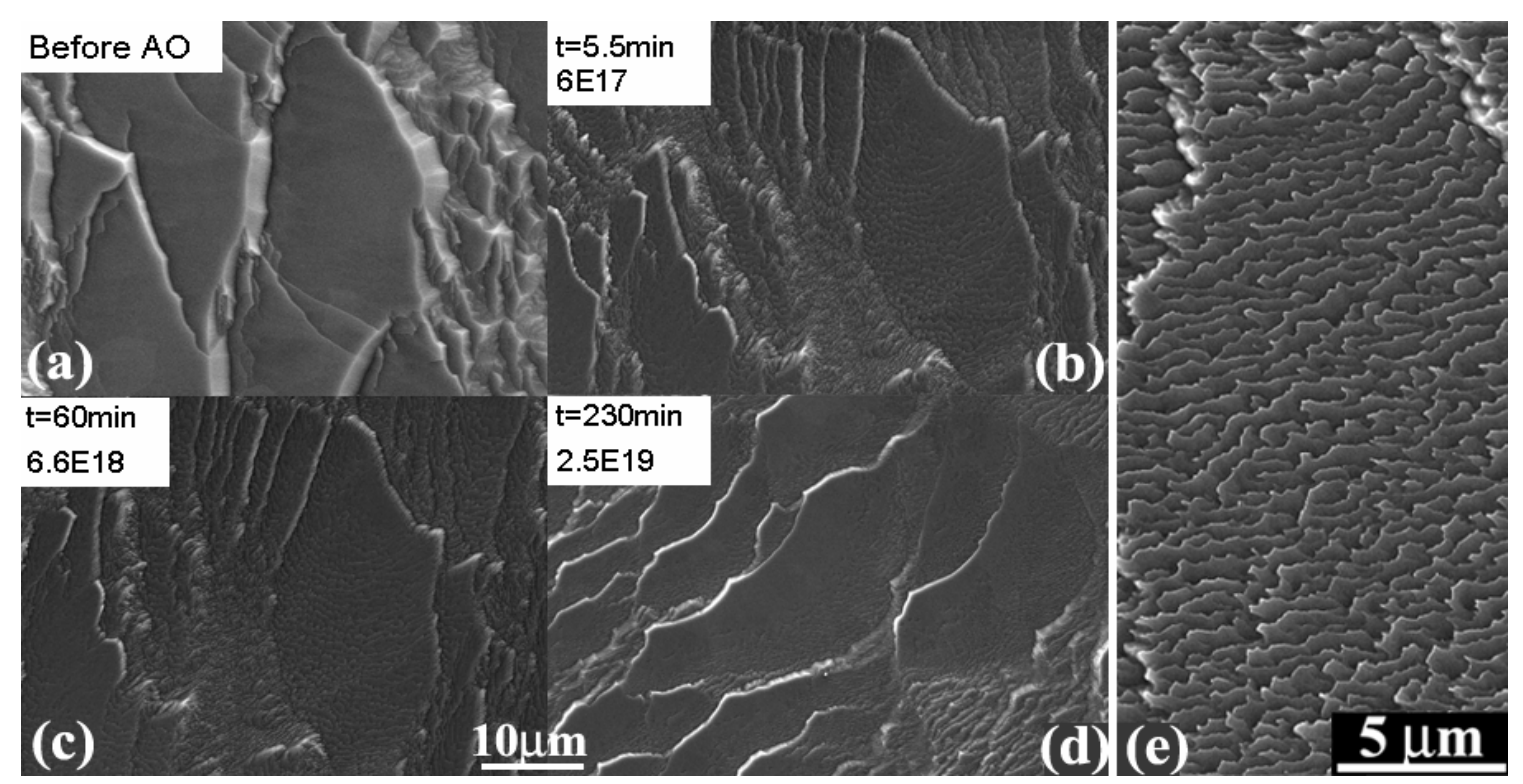

Fig. 2. A time sequence of SEM images revealing the surface evolution of $\mathrm{Al}(100)$ when it is exposed to $5 \mathrm{eV}$ atomic oxygen at room temperature. Fig.2 (e) magnified image of (b) where a significant surface roughening is noted. 